\title{
Gender analysis in the development and validation of FFQ: a systematic review
}

\author{
Hanna Lee ${ }^{1}$, Minji Kang ${ }^{2}$, Won Ok Song ${ }^{3}$, Jae Eun Shim ${ }^{4}$ and Hee Young Paik ${ }^{1,2 *}$ \\ ${ }^{1}$ Department of Food and Nutrition, Seoul National University, 1 Gwanak-ro, Gwanak-gu, Seoul o8826, Republic of Korea \\ ${ }^{2}$ Research Institute of Human Ecology, College of Human Ecology, Seoul National University, 1 Gwanak-ro, Gwanak-gu, Seoul \\ 08826, Republic of Korea \\ ${ }^{3}$ Department of Food Science and Human Nutrition, Michigan State University, 220 Trowbridge Rd, East Lansing, \\ MI 48824, USA \\ ${ }^{4}$ Department of Food and Nutrition, Daejeon University, 62 Daehak-ro, Dong-gu, Daejeon 34520, Republic of Korea
}

(Final revision received 22 December 2014 - Submitted 28 August 2015 - Accepted 29 September 2015 - First published online 11 December 2015)

\section{Abstract}

FFQ comprising food items, intake frequency categories and portion sizes have been used in large-scale observational studies to assess long-term dietary exposure. Although gender is an important influence on food choice and portion size, gender differences are not often analysed during FFQ development. This study investigated whether gender differences were considered sufficiently when developing FFQ, which affects the results of validation studies. A PubMed search using combinations of 'FFQ', 'Food Frequency Questionnaire', 'Validation' and 'Validity' identified 246 validation studies available in English, published between January 1983 and May 2014, which included healthy male and female adults. The development process of the 196 FFQ used in the 246 validation studies was examined. Of these, twenty-one FFQ (10.7\%) considered gender during item selection or portion size determination, and were therefore classified as gender specific (GS), but 175 (89.3\%) did not consider gender, and were classified as 'not gender specific (NGS)'. When the ratios between intake levels obtained using the FFQ and a reference method for energy and seven nutrients were compared between the GS group and the NGS group, more significant differences were observed in women than in men (four $v$. one nutrient). Intake of three nutrients was significantly underestimated in both sexes in the GS group. In the NGS group, nutrient intakes were significantly overestimated more often in women than in men (four $v$. one). These results indicate that not considering gender in FFQ development causes greater inaccuracy in dietary intake assessment in women than in men. Results of nutritional epidemiological studies should be re-evaluated for their validity, especially if the studies used NGS-FFQ.

Key words: Gender: FFQ: Validation: Development

FFQ are commonly used in nutritional epidemiological studies on diet and disease because of their ability to simultaneously measure the usual intake levels of multiple dietary components. These questionnaires are also very cost-effective as they can be selfadministered and can be read by computers ${ }^{(1)}$. FFQ are composed of a list of food items selected by researchers, a frequency category to determine usual consumption and a measure of portion size. FFQ respondents are asked to report the frequency of consumption and the amount of food regularly consumed. FFQ have large variations in design characteristics, including differences in the food items included and the portion size questions; such variability can greatly affect the responses and calculated intakes ${ }^{(2)}$.

A frequently used approach in developing an FFQ targeted to a specific population is to search for recent population-specific dietary data that can be used to determine the food items, portion sizes and nutrient database that should be included ${ }^{(3)}$. As men and women differ in their preference for food items and consumption amounts, it is reasonable to assume that the items and portion sizes selected for FFQ should reflect these gender differences, and furthermore that FFQ should be based on the target gender group. However, whether gender differences in food items and portion sizes are sufficiently accounted for during development of FFQ is not known. In addition, the use of gender-specific (GS) FFQ should be examined to determine whether overall results are affected. In FFQ validation studies, data from men and women are often pooled together to increase the sample size and to achieve sufficient statistical power to detect differences and/or associations ${ }^{(4)}$, but it is not clear how this pooling affects the conclusions for different gender groups. This study was conducted to investigate whether gender differences are factored into FFQ development studies, and whether FFQ gender specificity affects the results of validation studies.

Abbreviations: GS, gender specific; NGS, not gender specific.

* Corresponding author: Professor H. Y. Paik, fax +82 2880 8775, email hypaik@snu.ac.kr 


\section{Methods}

\section{Study selection}

To evaluate whether published FFQ were developed and validated with consideration of gender, the literature was searched for published validation studies, and the development and validation processes were then examined. A PubMed search using combinations of 'FFQ', 'Food Frequency Questionnaire', 'Validation' and 'Validity' yielded a total of 1164 articles published between 1983 and May 2014. We excluded studies that had been conducted with subjects of a single sex, studies that included subjects other than healthy adults, were review articles or articles published in languages other than English or were primary research articles that could not be found through web searches. The resulting 246 articles that included healthy adult men and women in the validation stage were used for this analysis (online Supplementary Appendix). Some FFQ were validated more than once; 196 unique FFQ were identified, and the detailed development procedures for the FFQ used in these studies were examined (online Supplementary Appendix). The study selection flowchart is shown in Fig. 1. A systematic review of the published study was performed according to the preferred reporting items for systematic reviews and meta-analyses statement ${ }^{(5)}$.

No restriction was placed on follow-up duration or type of reference dietary assessment method. Standard data-extraction forms were developed, and the following information was extracted from the studies: authors, titles, published year, country, study design, sex distribution, age of subjects, reference method, validated dietary factors, FFQ-estimated intake and reference method-estimated intake.

\section{Classification of development studies}

After reviewing the development procedures of 196 FFQ, FFQ were classified as 'gender specific' if gender was considered when selecting food items, portion sizes or both. All the remaining FFQ were classified as 'not gender-specific (NGS)' FFQ. Several studies used gender to select food items via

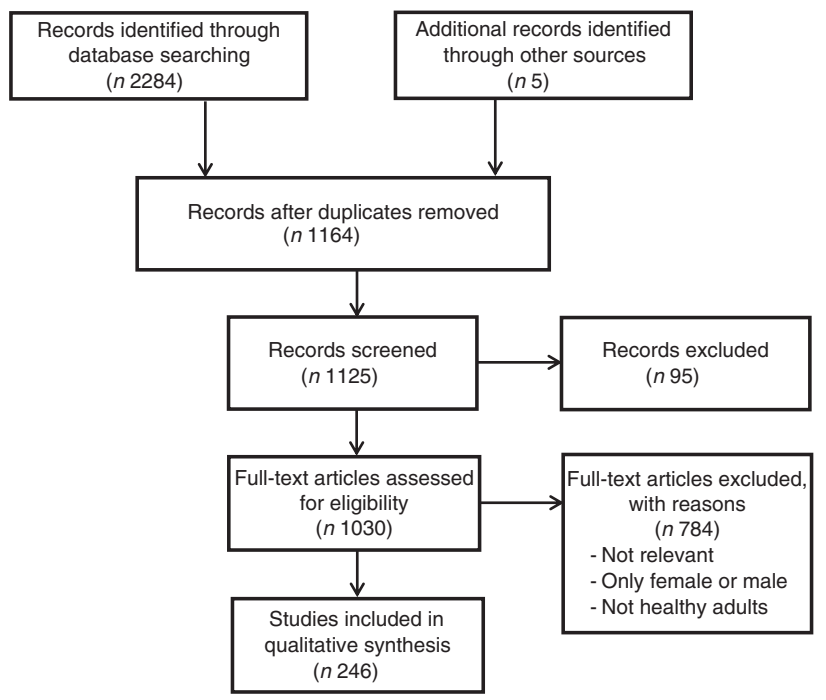

Fig. 1. The flowchart of literature search and study selection. multiple methods. In one study, sex was one of the factors used to help determine the foods omitted from the $\mathrm{FFQ}^{(6)}$. Another group performed step-wise multiple regression analyses to identify foods that are important in predicting individual nutrient intake, and these analyses were followed by additional procedures including the identification of foods and recipes by step-wise multiple regression analyses, conducted separately for men and women ${ }^{(7)}$. To determine portion sizes, one group used GS portions to calculate food intake in grams from the $\mathrm{FFQ}^{(8)}$. In another study, average portion sizes, according to gender and age derived from a national survey, were used ${ }^{(9)}$. The classification of studies into two groups was performed by two researchers, and in cases of disagreement the researchers discussed the studies until consensus was reached.

\section{Comparison of validation study results}

The 246 identified validation studies were divided into GS and NGS groups according to the gender specificity of the FFQ. If a validation study used a GS-FFQ, it was classified into the GS group. On the other hand, validation studies were classified into the NGS group if they were validating an NGS-FFQ. Nutrients used for validation in each study were identified, and eight nutrients reported in four or more studies in each group (energy, carbohydrate, protein, fat, cholesterol, fibre, Ca and vitamin C) were used to compare the validation results between the GS and NGS groups.

The mean intake levels of nutrients were extracted from each validation study. The ratio between the intake levels estimated by the FFQ and those estimated by the reference method in each study was compared for each nutrient. The mean ratio of ratios from individual studies for each nutrient was calculated using the following equation:

$$
\begin{gathered}
\text { Ratio }_{\text {study }}=\frac{\text { Nutrient intake estimated by FFQ }}{\text { Nutrient intake estimated by reference method }}, \\
\text { Mean ratio }=\frac{\text { Sum of ratio }_{\text {study }}}{\text { Number of studies }} .
\end{gathered}
$$

\section{Statistical analysis}

The characteristics of the selected FFQ development and validation studies are presented as numbers of studies and percentages. The mean nutrient intake levels reported in validation studies and intake ratios of selected nutrients estimated by the FFQ and by the reference methods are presented.

The difference in mean ratios between the GS group and the NGS group was assessed using Wilcoxon's test. Each mean ratio was compared using a one-sample $t$ test. All the statistical analyses were performed using Stata software package (version 13; StataCorp LP).

\section{Results}

Among the 196 studies describing the process of FFQ development, only twenty-one studies (10.7\%) considered gender and were therefore classified as GS-FFQ (see Table 1). Six of the GS-FFQ development studies included only female subjects. 
Table 1. Classification of FFQ according to whether gender was considered during development (Number of studies and percentages)

\begin{tabular}{|c|c|c|c|c|}
\hline \multirow[b]{2}{*}{ Classifications } & \multicolumn{2}{|r|}{ Gender consideration } & \multirow[b]{2}{*}{$n$} & \multirow[b]{2}{*}{$\%$} \\
\hline & Types & Method used & & \\
\hline \multirow[t]{4}{*}{ Gender specific } & Food item only & $\begin{array}{l}\text { Food lists for important sources of target nutrients were prepared for both genders } \\
\text { Information to evaluate gender specificity during determination of portion size was not available }\end{array}$ & 4 & $2 \cdot 0$ \\
\hline & Portion size only & $\begin{array}{l}\text { Gender differences in intake were reflected either in the determination of portion size or in } \\
\text { the nutrient database without portion size } \\
\text { Information to evaluate gender specificity in the selection of food items was not available }\end{array}$ & 10 & $5 \cdot 1$ \\
\hline & $\begin{array}{l}\text { Food item and } \\
\text { portion size }\end{array}$ & $\begin{array}{l}\text { Food lists for important sources of target nutrients were prepared for both genders } \\
\text { Gender differences in intake were reflected either in the determination of portion size or in } \\
\text { the nutrient database without portion size }\end{array}$ & 1 & 0.5 \\
\hline & $\begin{array}{l}\text { Single sex } \\
\text { (female only) }\end{array}$ & & 6 & $3 \cdot 1$ \\
\hline Not gender specific & & - & 175 & $89 \cdot 3$ \\
\hline Total & & & 196 & $100 \cdot 0$ \\
\hline
\end{tabular}

More studies considered gender as a factor when determining portion size rather than for selecting the food items to be included in the FFQ (eleven and five studies, respectively).

The validation studies used in the analysis are presented in Table 2. The studies are classified into GS and NGS groups according to the gender specificity of the FFQ used. Eight different GS-FFQ and twenty-one different NGS-FFQ were used in nine GS and twenty-six NGS studies, respectively. Four studies in the NGS group included fewer than 100 subjects. The characteristics of the studies are summarised in Table 2.

The mean intake levels of energy and seven nutrients estimated by the FFQ and by the reference method used in each validation study - and the ratios of intake levels obtained by the two methods are shown in Table 3 .

The ratio between the intake data obtained by the FFQ compared with the reference method ranged from 0.83 to 1.15 for the GS group in men and from 0.82 to 1.14 in women, whereas the ratio in the NGS group ranged from 0.95 to 1.21 in men and from 1.05 to 1.36 in women. For the GS group, the mean ratios for energy, protein and fat were significantly $<1$ in both sexes. In comparison, in the NGS group, the ratios for all nutrients except vitamin $\mathrm{C}$ were not significantly different from 1 among men, whereas the ratios for energy, carbohydrate, $\mathrm{Ca}$ and vitamin $\mathrm{C}$ were significantly different from 1 among women. Vitamin $\mathrm{C}$ intake tended to be overestimated in both the GS and NGS groups, but more so in the NGS group in both sexes. This result indicates that NGS-FFQ overestimate the intake of energy and several nutrients in women, but not in men, whereas GS-FFQ may underestimate some nutrients similarly in both sexes.

\section{Discussion}

Over the past several decades, non-communicable diseases (NCD) have increased worldwide and are now a leading cause of death. Four behavioural factors - tobacco use, insufficient physical activity, harmful use of alcohol and an unhealthy diet have been actively investigated in the aetiology and management of $\mathrm{NCD}^{(10)}$. FFQ have been widely used to estimate the usual intake levels of dietary constituents for studies on dietdisease relationships. As participants respond according to the items listed and portion sizes presented in the FFQ, the selection of food items and their associated portion sizes is crucial for accurate assessment of dietary factors.

Gender differences in food preferences and consumption amounts are easily observed. Bates et al. ${ }^{(11)}$ suggested that women eat better overall and consume more full-fat milk, certain beverages, cakes, apples, pears and bananas, whereas men eat more eggs, sugar, certain meat products and drink more alcoholic drinks, especially beer and lager. A study by Pollard et $a l^{(12)}$ reported that men ate more meat and less fruit than women, and that there was strong evidence that these gender differences were due to women being more concerned with weight control, food naturalness and ethical issues ${ }^{(10)}$. Johansson et $a l^{(13)}$ also suggested that gender differences exist in the reporting of energy intake: in both their own and another national study, women were more likely to under-report energy intake.

Men usually eat more food than women, but actual portion size differences vary by food item. Cade et $a l^{(14)}$ described gender differences in food portion sizes, and recommended the use of sex-specific 'typical' portion weights instead of 'standard' portions to estimate nutrient intake from food frequency intake data. However, little attention has been given to gender differences in food choices and consumption amounts during FFQ development, or to how these gender differences may affect the survey results.

This systematic review focused on how gender differences were considered during FFQ development, and how gender specificity affected the results of FFQ validation studies. Among the 196 FFQ development studies found in the literature, gender differences were factored into only $10 \%$ of the studies. Gender differences were more often considered when determining portion sizes than for food item selection. As the occurrence of many diseases (and their risk/preventive factors) varies by gender, it is not certain whether validation without proper design or analysis according to gender is appropriate for detecting diet-disease relationships. A comparison between the portion sizes of men and women in a previously developed FFQ revealed that actual portion sizes were larger for $84 \%$ of items; furthermore, for $73 \%$ of items, men had a larger average portion size than women (Noh et al unpublished results). In this study, when FFQ were corrected for differences in portion size between men and women, and the results of the validation studies were re-calculated, intake levels of 


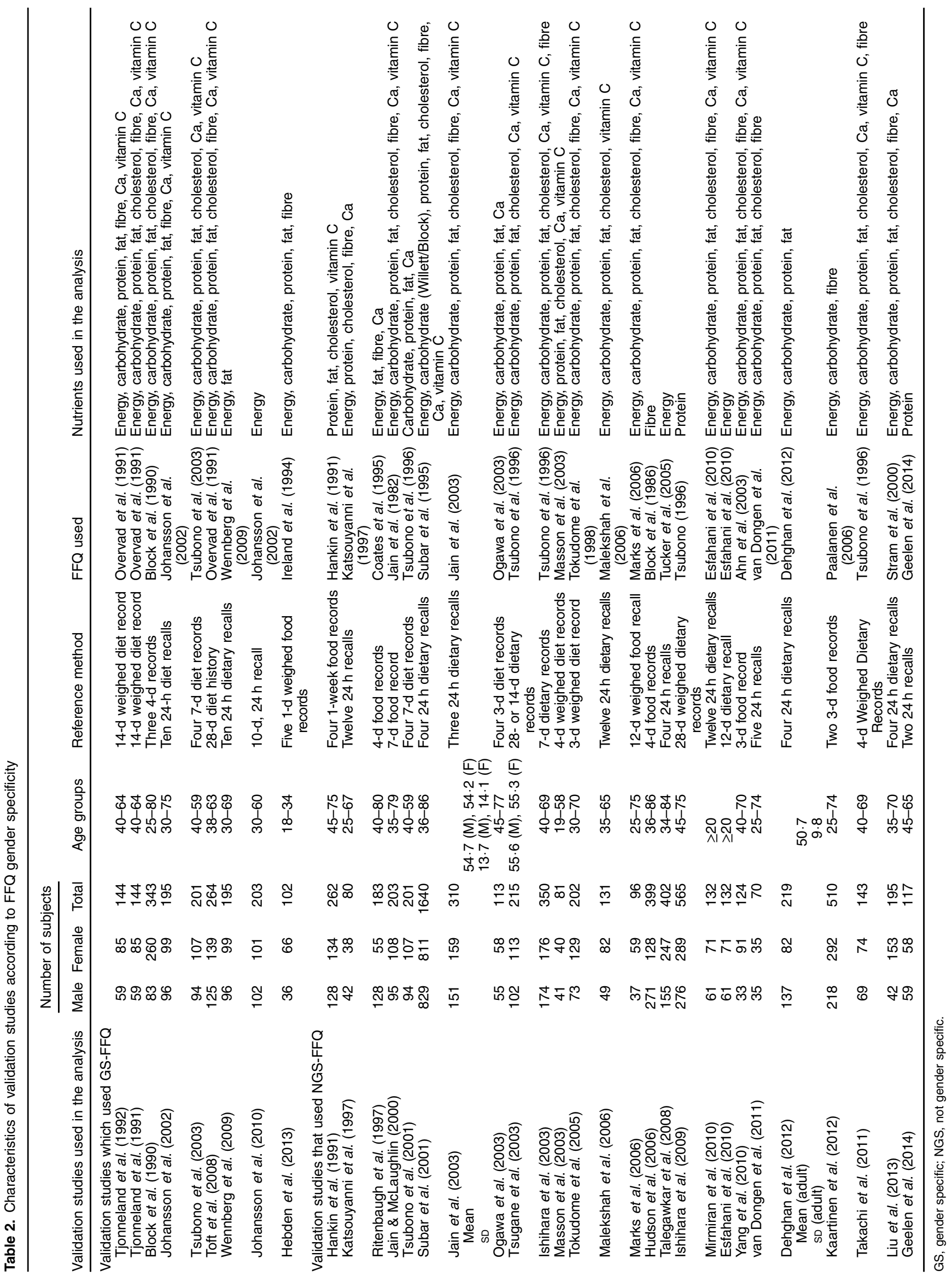


Table 3. Comparison of estimated mean energy and nutrient intake ratios between FFQ:reference methods according to FFQ gender specificity

\begin{tabular}{|c|c|c|c|c|c|c|c|c|c|c|}
\hline \multirow[b]{3}{*}{ Nutrients } & & & \multicolumn{4}{|c|}{ GS group } & \multicolumn{4}{|c|}{ NGS group } \\
\hline & \multicolumn{2}{|c|}{ Number of studies } & \multirow[b]{2}{*}{ Reference method $(A)$} & \multirow[b]{2}{*}{ FFQ (B) } & \multicolumn{2}{|l|}{ Ratio } & \multirow[b]{2}{*}{ Reference method $(\mathrm{A})$} & \multirow[b]{2}{*}{$F F Q(B)$} & \multicolumn{2}{|l|}{ Ratio } \\
\hline & GS & NGS & & & Ratio (B:A) & $\mathrm{SD}_{\text {ratio }}$ & & & Ratio (B:A) & $\mathrm{SD}_{\text {ratio }}$ \\
\hline \multicolumn{11}{|l|}{ Men } \\
\hline Energy (kJ) & 38 & 88 & $9764 \cdot 2$ & $8997 \cdot 7$ & 3.85 & 0.29 & $9616 \cdot 5$ & $9777 \cdot 6$ & $4 \cdot 27$ & 0.63 \\
\hline Energy (kcal) & 9 & 21 & 2333.7 & $2150 \cdot 5$ & $0.92 \dagger$ & 0.07 & 2298.4 & $2336 \cdot 9$ & 1.02 & 0.15 \\
\hline Carbohydrate (g) & 7 & 17 & $251 \cdot 7$ & $255 \cdot 2$ & $1 \cdot 01$ & $0 \cdot 10$ & $302 \cdot 1$ & $311 \cdot 2$ & 1.03 & $0 \cdot 16$ \\
\hline Protein $(\mathrm{g})$ & 7 & 21 & $90 \cdot 7$ & $79 \cdot 6$ & $0.88 \dagger$ & $0 \cdot 10$ & $87 \cdot 9$ & $83 \cdot 1$ & 0.95 & 0.20 \\
\hline Fat $(g)^{*}$ & 8 & 19 & $91 \cdot 1$ & $77 \cdot 6$ & $0.85 \ddagger$ & $0 \cdot 14$ & $70 \cdot 7$ & 71.5 & 1.01 & 0.29 \\
\hline Cholesterol (mg) & 4 & 16 & $391 \cdot 7$ & $325 \cdot 8$ & 0.83 & $0 \cdot 17$ & $304 \cdot 8$ & $289 \cdot 0$ & 0.95 & 0.26 \\
\hline Fibre $(\mathrm{g})$ & 5 & 14 & $21 \cdot 2$ & $22 \cdot 4$ & 1.06 & 0.07 & $21 \cdot 0$ & $22 \cdot 0$ & 1.05 & 0.35 \\
\hline $\mathrm{Ca}(\mathrm{mg})$ & 6 & 16 & $1065 \cdot 2$ & $1044 \cdot 3$ & 0.98 & $0 \cdot 16$ & $778 \cdot 4$ & $797 \cdot 4$ & 1.02 & 0.22 \\
\hline Vitamin C (mg) & 6 & 13 & 88.5 & $101 \cdot 8$ & $1 \cdot 15$ & 0.35 & $116 \cdot 9$ & 141.4 & $1 \cdot 21 \dagger$ & 0.26 \\
\hline Median & & & & & 0.95 & $0 \cdot 12$ & & & 1.02 & 0.24 \\
\hline Median (vitamin C excluded) & & & & & 0.92 & $0 \cdot 10$ & & & 1.02 & 0.22 \\
\hline \multicolumn{11}{|l|}{ Women } \\
\hline Energy (kJ) & 38 & 88 & $7112 \cdot 4$ & $6503 \cdot 2$ & $3 \cdot 81$ & 0.46 & $7304 \cdot 8$ & $8017 \cdot 4$ & $4 \cdot 60$ & 0.84 \\
\hline Energy $(\mathrm{kcal})^{\star *}$ & 9 & 21 & $1699 \cdot 9$ & $1554 \cdot 3$ & $0.91 \dagger$ & 0.11 & 1745.9 & $1916 \cdot 2$ & $1 \cdot 10 \dagger$ & 0.20 \\
\hline Carbohydrate (g) & 7 & 17 & $192 \cdot 4$ & $192 \cdot 1$ & 1.00 & 0.21 & $237 \cdot 2$ & $262 \cdot 4$ & $1.11 \dagger$ & 0.23 \\
\hline Protein $(\mathrm{g})^{\star}$ & 7 & 21 & $68 \cdot 2$ & $60 \cdot 2$ & $0.88 \dagger$ & $0 \cdot 10$ & $67 \cdot 8$ & $72 \cdot 9$ & 1.08 & 0.26 \\
\hline Fat $(\mathrm{g})^{\star \star}$ & 8 & 19 & $66 \cdot 8$ & $54 \cdot 8$ & $0.82 \ddagger$ & $0 \cdot 10$ & 55.5 & 62.8 & $1 \cdot 13$ & 0.27 \\
\hline Cholesterol (mg) & 4 & 16 & 323.3 & 285.9 & 0.88 & 0.30 & $235 \cdot 3$ & 246.9 & 1.05 & 0.31 \\
\hline Fibre $(\mathrm{g})$ & 6 & 14 & $15 \cdot 7$ & $17 \cdot 8$ & $1 \cdot 14$ & 0.37 & $17 \cdot 4$ & $20 \cdot 8$ & $1 \cdot 21$ & 0.32 \\
\hline $\mathrm{Ca}(\mathrm{mg})^{*}$ & 6 & 16 & $887 \cdot 0$ & $849 \cdot 8$ & 0.96 & 0.09 & $669 \cdot 3$ & $766 \cdot 6$ & $1 \cdot 15 \ddagger$ & 0.27 \\
\hline Vitamin C (mg) & 6 & 13 & 92.5 & $102 \cdot 9$ & $1 \cdot 11$ & 0.36 & $110 \cdot 5$ & $150 \cdot 3$ & $1.36 \neq$ & 0.30 \\
\hline Median & & & & & 0.93 & 0.16 & & & $1 \cdot 12$ & 0.27 \\
\hline Median (vitamin C excluded) & & & & & 0.91 & 0.11 & & & $1 \cdot 11$ & 0.27 \\
\hline
\end{tabular}

GS, gender specific; NGS, not gender specific.Mean ratios differed significantly between the GS and NGS groups on the Wilcoxon's test: ${ }^{*} P<0.05,{ }^{\star *} P<0.01$.

Mean ratio was significantly different from 1 with the one-sample $t$ test: $\dagger P<0.05, \ddagger P<0.01$.

energy and other nutrients including fat changed significantly. The direction of change differed by gender. Energy intake increased significantly in men but not in women, whereas fat intake increased in both genders. Portion size variations differ between men and women. Tsubono et $a l^{(15)}$ analysed within- and between-person variability in adult Japanese subjects. For $58 \%$ of forty-five food items, all of which were consumed with sufficient frequency, the ratio was larger in men (twenty-six of forty-five items). Therefore, there should be more careful consideration when determining portion sizes for men and women.

The results of this study indicate that NGS-FFQ that fail to consider the difference in portion size between women and men may overestimate nutrient intake of women compared with the levels obtained using reference methods. In comparison, the overall nutrient intake of men estimated using NGSFFQ was similar to the estimates obtained using reference methods. These results seem to be partially attributable to the fact that the NGS-FFQ and reference methods exhibited common measurement errors in men. Compared with women, men were more likely to report difficulties in recalling what and how much they ate during the dietary survey ${ }^{16}$. In contrast, estimated intake using GS-FFQ had a similar pattern, irrespective of whether it was in men or in women. As a result, the affected nutrients and the degree to which they were overestimated differed between men and women, depending on the type of FFQ used: fat in men and energy, protein, fat and Ca in women. Therefore, subjects could be misclassified such that risk association is attenuated. Although many studies have been conducted on diet-disease relationships, gender-related effects on dietary assessment tools have seldom been investigated. Our study is the first to assess the performance of FFQ according to whether gender was considered during the development stage. A literature review on the design, utilisation and validation of FFQ using a semi-systematic approach was published in 2004, but it focused on the overall characteristics of 227 validation studies and 164 utilisation studies, not on gender specifically ${ }^{(14)}$. Failure to consider gender during the development of FFQ resulted in general overestimation of the energy and nutrient intakes of women compared with men, whereas the errors tended to be similar in men and women for GS-FFQ. As $89 \%$ of FFQ were developed between 1983 and 2014, it is reasonable to assume that most of the cohort studies in nutritional epidemiology conducted in recent decades have used NGS-FFQ. On the basis of the results of the present study that estimation of dietary intakes obtained using NGS-FFQ tends to be less accurate, and more importantly errors are larger in women, validity of the results on diet-disease relationships from these studies are questionable, especially in studies conducted in women and in subjects of both sexes. Results of the studies that used NGS-FFQ should be critically re-evaluated for their validity of dietary information and the association of dietary factors and disease outcomes, with special concerns given to gender differences.

Our findings are limited because of the insufficient number of comparable studies, particularly due to the small number of GS-FFQ that have been developed and the small number of 
validation studies that have used them. In addition, the validation studies differed in terms of the reference method used and the age and other characteristics of the study subjects, but we could not include these differences in the analysis. We only included journal articles published in English and those that included healthy men and women. Due to the limited number of GS-FFQ, whether gender was considered in selecting food items, determining portion sizes or both was not assessed during comparison of the validation study results. More studies are required to further assess the effects of GS food items and portion size selection, because each of these two factors is essential in the composition of FFQ.

\section{Acknowledgements}

None.

This research received no specific grant from any funding agency, commercial or not-for-profit sectors.

H. L., M. K., W. O. S., J. E. S. and H. Y. P.: designing the study; H. L., M. K., H. Y. P. and J. E. S.: carrying it out; H. L., H. Y. P., W. O. S. and J. E. S.: writing the article; H. L. and H. Y. P.: analysing the data; W. O. S., J. E. S. and H. Y. P.: formulating the research question(s).

The authors declare that there are no conflicts of interest.

\section{Supplementary material}

For supplementary material/s referred to in this article, please visit http://dx.doi.org/doi:10.1017/S0007114515004717

\section{References}

1. Hebden L, Kostan E, O'Leary F, et al. (2013) Validity and reproducibility of a food frequency questionnaire as a measure of recent dietary intake in young adults. PLOS ONE 8, e75156.

2. Molag ML, de Vries JH, Ocké MC, et al. (2007) Design characteristics of food frequency questionnaires in relation to their validity. Am J Epidemiol 166, 1468-1478.
3. Subar AF (2004) Developing dietary assessment tools. J Am Diet Assoc 104, 769-770.

4. Jacobs ET, Thompson PA \& Martinez ME (2007) Diet, gender, and colorectal neoplasia. J Clin Gastroenterol 41, 731-746.

5. Moher D, Liberati A, Tetzlaff J, et al. (2009) Preferred reporting items for systematic reviews and meta-analyses: the PRISMA statement. PLoS Med 6, e1000097.

6. Block G, Hartman AM \& Naughton D (1990) A reduced dietary questionnaire: development and validation. Epidemiology $\mathbf{1}$, 58-64.

7. Overvad K, Tjønneland A, Haraldsdóttir J, et al. (1991) Development of a semiquantitative food frequency questionnaire to assess food, energy and nutrient intake in Denmark. Int J Epidemiol 20, 900-905.

8. Eysteinsdottir T, Thorsdottir I, Gunnarsdottir I, et al. (2012) Assessing validity of a short food frequency questionnaire on present dietary intake of elderly Icelanders. Nutr J 11, 12.

9. Johansson I, Hallmans G, Wikman A, et al. (2002) Validation and calibration of food-frequency questionnaire measurements in the Northern Sweden Health and Disease cohort. Public Health Nutr 5, 487-496.

10. World Health Organization (2010) Global Status Report on Non-Communicable Diseases 2010. Geneva: WHO.

11. Bates CJ, Prentice A \& Finch S (1999) Gender differences in food and nutrient intakes and status indices from the National Diet and Nutrition Survey of people aged 65 years and over. Eur I Clin Nutr 53, 694-699.

12. Pollard TM, Steptoe A \& Wardle J (1998) Motives underlying healthy eating: using the Food Choice Questionnaire to explain variation in dietary intake. J Biosoc Sci 30, 165-179.

13. Johansson L, Solvoll K, Bjørneboe GE, et al. (1998) Underand overreporting of energy intake related to weight status and lifestyle in a nationwide sample. Am J Clin Nutr 68 , 266-274.

14. Cade JE, Burley VJ, Warm DL, et al. (2004) Food-frequency questionnaires: a review of their design, validation and utilization. Nutr Res Rev 17, 5-22.

15. Tsubono Y, Kobayashi M, Takahashi T, et al. (1997) Withinand between-person variations in portion sizes of foods consumed by the Japanese population. Nutr Cancer 29 , $140-145$.

16. Kang H, Jung HJ \& Paik HY (2009) Analysis of foods and nutrients intake obtained at the final probing step in 24-hour recall method (in Korean). Korean J Nutr 42, 158-170. 